\title{
A tool to predict survival in stage IV entero-pancreatic NEN
}

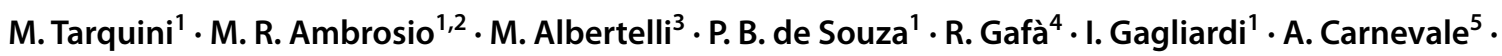 \\ P. Franceschetti ${ }^{2} \cdot$ M. C. Zatelli ${ }^{1,2}$ (D)
}

Received: 27 July 2020 / Accepted: 22 August 2020 / Published online: 6 September 2020

(c) The Author(s) 2020

\begin{abstract}
Purpose Well-differentiated stage IV neuroendocrine neoplasms (NEN) have an extremely heterogeneous, unpredictable clinical behavior. Survival prognostic markers, such as the recently proposed NEP-Score, would be very useful for better defining therapeutic strategies. We aim to verify NEP-Score applicability in an independent cohort of stage IV well-differentiated (WD) gastroentero-pancreatic (GEP) NEN, and identify a derivate prognostic marker taking into account clinical and pathological characteristics at diagnosis.

Methods Age, site of primary tumor, primary tumor surgery, symptoms, Ki67, timing of metastases of 27 patients (10 females; mean age at diagnosis $60.2 \pm 2.9$ years) with stage IV WD GEP NEN were evaluated to calculate the NEP-Score at the end of follow-up (NEP-T). We calculated the NEP-Score at diagnosis (NEP-D), which does not consider the appearance of new metastases during follow-up. Patients were subdivided according to whether they were alive or not at the end of follow-up (EOF) and an NEP-Score threshold was investigated to predict survival.

Results Mean NEP-T and mean NEP-D were significantly lower in 15 live patients as compared to 12 deceased patients $(p<0.01)$ at EOF. We identified an NEP-D $=116$ as the cutoff that significantly predicts survival. No gender differences were identified.

Conclusions In our series, we confirmed NEP-Score applicability. In addition, we propose NEP-D as a simple, quick and cheap prognostic score that can help clinicians in decision making. NEP-D threshold can predict NEN aggressiveness and may be used to define the best personalized therapeutic strategy.
\end{abstract}

Keyword Neuroendocrine neoplasms $\cdot$ NEP-Score $\cdot$ NEP-D $\cdot$ NEP-T $\cdot$ Survival

Electronic supplementary material The online version of this article (https://doi.org/10.1007/s40618-020-01404-4) contains supplementary material, which is available to authorized users.

\section{C. Zatelli}

ztlmch@unife.it

1 Department of Medical Sciences, Section of Endocrinology and Internal Medicine, University of Ferrara, Via Ariosto 35, 44100 Ferrara, Italy

2 Endocrine Unit, Azienda Ospedaliero-Universitaria di Ferrara, Via Aldo Moro 8, Cona, 44124 Ferrara, Italy

3 Endocrinology, Department of Internal Medicine DiMI, University of Genova, Genoa, Italy

4 Pathology Unit, Department of Medical Sciences, University of Ferrara, Ferrara, Italy

5 Department of Morphology, Surgery and Experimental Medicine, University of Ferrara, Ferrara, Italy

\begin{tabular}{ll}
\multicolumn{2}{l}{ Abbreviations } \\
WD & Well differentiated \\
OS & Overall survival \\
GEP NEN & $\begin{array}{l}\text { Gastroentero-pancreatic neuroendocrine } \\
\text { neoplasms }\end{array}$ \\
NEP-Score & Neuroendocrine prognostic score \\
EOF & End of follow-up
\end{tabular}

\section{Introduction}

Epidemiological studies indicate that neuroendocrine neoplasms (NEN) are rare diseases, with a low incidence that increased over time in the last years [1-4]. Thanks to the improved therapeutic possibilities for NEN patients, prevalence is also increasing, being associated with different clinical outcomes according to grade, stage, age at diagnosis, and primary site of the tumor [1, 4-12]. Therapeutic management of gastroentero-pancreatic (GEP) NEN is 
mainly based on tumor differentiation and grading [5-7, 13-15], but markers of clinical outcome that could predict patient survival are still lacking [16]. Despite improvements in prognostic grading and staging systems, the challenge to predict outcome for patients with GEP NEN is difficult, particularly in stage IV GEP NEN, since these patients are often metastatic at the time of diagnosis and may display a wide spectrum of clinical behavior, ranging from indolent to aggressive, even within apparently homogeneous categories. Thus, selecting the optimal treatment is a challenging task. Blood-based biomarkers, such as chromogranin A ( $\mathrm{CgA})$ $[8,9,12,17,18]$, circulating tumor cells and microRNAs [19], as well as tissue markers [20, 21] have been considered, but these putative markers have not been fully validated, so far. Pusceddu and co-workers [22] developed a classification prognostic score for overall survival (OS) in patients with stage IV well-differentiated (WD) G1-G2 NEN, named NEP-Score (NEuroendocrine Prognostic Score) by evaluating a monocentric cohort of patients with a long follow-up as training set (providing data reliability and homogeneity) and then two independent validation cohorts [8]. By using this score, they identified three different groups in terms of survival at 10 years: patients at low risk (NEP-Score $\leq 70$ ) displayed $>70 \%$ survival; patients at intermediate risk (70 < NEP-Score $\leq 198)$ displayed 30-70\% survival; patients at high risk (NEP-Score $\geq 199$ ) displayed $<30 \%$ survival. In their study, NEP-Score turned out to be useful to rank patients according to their risk of death, predicting OS. The aim of the present study is to verify the applicability of the NEP-Score to an independent cohort of stage IV WD enteropancreatic NEN, and to identify a derivate marker capable of predicting patients prognosis by taking into account clinical and pathological characteristics at diagnosis.

\section{Methods}

\section{Study design}

In this observational study, we retrospectively evaluated NEP-Score in a series of entero-pancreatic NEN patients referring to our center from 2008 to 2018 . In line with previous studies [22], exclusively stage IV WD entero-pancreatic NEN patients with a follow-up $>24$ months were included, while, as previously reported, patients with poorly differentiated neuroendocrine carcinomas (NEC G3) were excluded.

\section{Patients}

We selected a group of 27 patients ( 10 females, 17 males; mean age at the diagnosis $60.2 \pm 2.9$ years; median age at diagnosis $=64$ years; range $26-84$ years) $($ Table 2 ) with stage IV WD entero-pancreatic NEN, characterized according to
Table 1 NEP-Score calculation (modified from Reference [22])

\begin{tabular}{ll}
\hline & Score \\
\hline Age & \\
$<45$ & 0 \\
$46-65$ & 28 \\
$>65$ & 58 \\
Site of primary tumor & \\
Ileum & 0 \\
Pancreas & 59 \\
Primary tumor surgery & \\
Yes & 0 \\
No & 100 \\
Functional status & \\
Yes & 32 \\
No & 0 \\
Ki67 & \\
$0-2$ & \\
$3-20$ & 0 \\
$>20$ & 12 \\
Timing of metastases & 57 \\
Synchronous & \\
Metachronous $>24$ months & \\
Metachronous $\leq 24$ months & \\
\hline
\end{tabular}

Table 2 Patients' general features

\begin{tabular}{|c|c|c|}
\hline & number & $\%$ \\
\hline \multicolumn{3}{|l|}{ Gender } \\
\hline Males & 17 & 63 \\
\hline Females & 10 & 37 \\
\hline \multicolumn{3}{|c|}{ Primary tumor site } \\
\hline Ileum & 13 & 48 \\
\hline Pancreatic & 14 & 52 \\
\hline \multicolumn{3}{|c|}{ Metastasis timing } \\
\hline Synchronous & 12 & 44 \\
\hline Metachronous & 15 & 56 \\
\hline \multicolumn{3}{|l|}{ Ki67 (Mib-1) } \\
\hline $0-2$ & 17 & 63 \\
\hline $3-20$ & 7 & 26 \\
\hline$>20$ & 3 & 11 \\
\hline \multicolumn{3}{|l|}{ Functional status } \\
\hline No & 18 & 67 \\
\hline Yes & 9 & 33 \\
\hline \multicolumn{3}{|c|}{ Primary tumor surgery } \\
\hline No & 6 & 22 \\
\hline Yes & 21 & 78 \\
\hline \multicolumn{3}{|l|}{ Alive at EOF } \\
\hline No & 12 & 44 \\
\hline Yes & 15 & 56 \\
\hline
\end{tabular}


the 2019 WHO classification of tumors of the digestive system. Among these patients, 55\% patients had documented progressive disease at the time of score calculation at diagnosis. All of them had been treated with long-acting somatostatin analogs and two patients with insulinoma had been treated with everolimus. Patients were evaluated for the following characteristics to calculate the NEP-Score at the end of follow-up (NEP-T) which lasted $70.3 \pm 11.6$ months: age, site of primary tumor, primary tumor surgery, symptoms, grading, timing of metastases, assigning the respective scores (see Table 1). A modified NEP-Score was then calculated, considered as the NEP-Score at diagnosis (NEP-D), which does not take into account the appearance of new metastases during follow-up. Patients were subdivided according to whether they were alive or not at the end of follow-up (EOF). Patient characteristics are displayed in Table 2 and in Supplemetary Table 1. This study is in accordance with the principles set out in the Declaration of Helsinki, has been specifically approved by the Local Ethics Committee (Comitato Etico Indipendente di Area Vasta Emilia Centro, CE-AVEC, at the Policlinico S.Orsola-Malpighi in Bologna) and authorized by the General Director of the Azienda Ospedaliero Universitaria in Ferrara (protocol number CE-AVEC 238/2020/Oss/AOUFe). Written consent was obtained from each patient or subject after full explanation of the purpose and nature of all procedures used.

\section{Statistical evaluation}

Categorical data were summarized using frequencies and percentages. The Chi-square $\left(\chi^{2}\right)$ test was performed to evaluate the presence of statistically significant differences among the evaluated groups in terms of NEP-Score. The paired Student's $t$ test was employed to compare the mean NEP-D and NEP-T scores among groups. A $p$ value $<0.05$ was considered significant. Sensitivity, specificity, positive predictive value (PPV), negative predictive value (NPV), and accuracy were calculated for each identified NEP-D and NEP-T threshold. All statistical analyses were performed using GraphPad software.

\section{Results}

\section{NEP-T score calculation}

In our series, we found a mean NEP-T, corresponding to the original NEP-Score, of $145.5 \pm 16.5$. Patients were then subdivided according to whether they were alive or not at the EOF. We found a significant difference between mean NEP-T in live patients $(100.3 \pm 17.1 ; 15$ patients $)$ as compared to deceased patients at EOF $(202.1 \pm 21.3 ; 12$ patients; $p<0.01$ ) (Fig. 1).

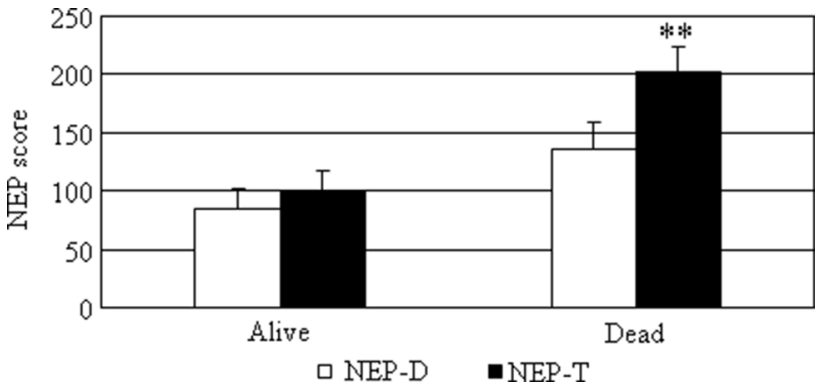

Fig. 1 NEP-T and NEP-D scores. NEP-T (black columns) and NEP-D (white columns) scores are expressed as mean \pm standard error of the mean (SEM). ${ }^{* *} p<0.01$ dead vs. alive patients at the end of followup

\section{NEP-D score calculation}

A modified NEP-Score was then calculated, considered as the NEP-Score at diagnosis (NEP-D), which does not take into account the appearance of new metastases during follow-up. In our series, we found a mean NEP-D of $108.0 \pm 14.0$. Patients were subdivided according to whether they were alive or not at EOF. We found that mean NEP-D in live patients $(85.6 \pm 16.7)$ was lower as compared to deceased patients $(136.1 \pm 22.5)$ at EOF, but this difference did not reach statistical significance (Fig. 1).

\section{Gender}

We also considered a possible difference between genders. In male patients, mean NEP-T of the eight patients (mean age $=70.6 \pm 2.8$ years) who were alive at EOF was $69.5 \pm 14.8$, while the mean NEP-T of the nine patients (mean age $=64.3 \pm 3.0$ years) who were dead at EOF was significantly higher $(203.3 \pm 26.7 ; p<0.01)$. This difference was not correlated to aging, since mean age at last followup in the two groups was not significantly different. Concerning female patients, mean NEP-T of the seven patients (mean age $=60.1 \pm 2.6$ years) who were alive at EOF was $135.4 \pm 30.2$, which was similar $(198.3 \pm 37.6 ; p=$ not significant) to the mean NEP-T of the three patients (mean age $=73.3 \pm 1.7$ years) who were dead at EOF. Similarly to males, there was no significant difference between the mean age of the two groups. Comparing males and females, we found that NEP-T in live patients was significantly lower in males as compared to females $(p<0.05)$. In addition, at EOF, $30 \%$ of female patients ( 3 out of 10 ) vs. $52.3 \%$ of male patients ( 8 out of 17) were dead. The difference in the proportion of live patients according to gender did not reach statistical significance. On the other hand, NEP-T in dead patients was similar in males and females, in keeping with a similar overall survival $(\mathrm{OS}=51.6 \pm 18.9$ vs. $35.5 \pm 10.8$ months; $p=$ not significant). 
Concerning NEP-D, in male patients, mean NEP-D of live patients (mean age $=62.8 \pm 4.7$ years) was $55.8 \pm 7.0$, while the mean NEP-D of the patients who were dead at EOF (mean age $=60.0 \pm 3.9$ years) was significantly higher $(139.3 \pm 28.4 ; p<0.01)$. Again, this difference was not correlated to age, since mean age at diagnosis in the two groups was not significantly different. Concerning female patients, mean NEP-D of live patients (mean age $=53.3 \pm 8.8$ years) at EOF was $119.7 \pm 29.5$, being similar to the mean NEP-D of patients (mean age $=70.3 \pm 5.4$ years) who were dead at EOF $(126.3 \pm 37.6 ; p=$ not significant). Similarly to males, there was no significant difference between the mean age of the two groups. Comparing males and females, we found that NEP-D in live patients was significantly lower in males as compared to females $(p<0.05)$. On the other hand, NEP-D in dead patients was similar in males and females, as reported for NEP-T.

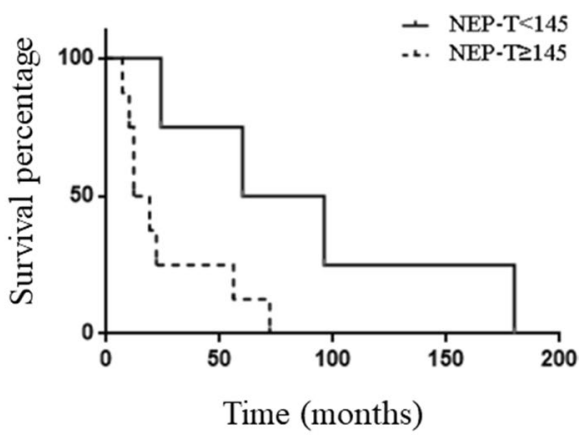

$\begin{array}{lccccc}\text { Number at NEP-T }<145 & 17 & 16 & 14 & 14 & 13 \\ \text { Number at NEP-T } \geq 145 & 10 & 4 & 2 & 2 & 2\end{array}$

Fig. 2 Patients' survival according to the NEP-T threshold. KaplanMeier curves for survival of 27 patients with stage IV WD GEP NEN according to NEP-T threshold $=145$

A

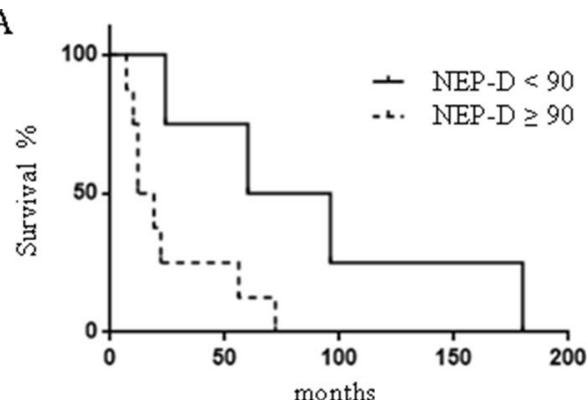

$\begin{array}{lccccc}<90 & 14 & 13 & 11 & 11 & 10 \\ \geq 90 & 13 & 7 & 5 & 5 & 5\end{array}$

Fig. 3 Patients' survival according to different NEP-D thresholds. Kaplan-Meier curves for survival of 27 patients with stage IV WD GEP NEN according to the NEP-D threshold $=90$ (a) and NEP-D threshold $=116$ (b). a Survival curve of patients with NEP-D $<90$

\section{NEP-T threshold}

A NEP-T score threshold was investigated to assess the reliability of this score in detecting disease status. We found that a NEP-T score threshold $\geq 145$ could correctly differentiate patients alive from those dead at EOF (Fig. 2). Indeed, $80 \%$ of patients with NEP-T $\geq 145$ and $23.5 \%$ of patients with NEP-T $<145$ were dead at EOF. This difference was statistically significant $(p<0.005)$. Similarly, OS was 3.5-fold shorter in patients with NEP-T $\geq 145$ as compared to those with NEP-T $<145$ ( $\mathrm{p}<0.05)$. A NEP-T $\geq 145$ value represents a cutoff, allowing the best compromise between sensitivity $(67 \%)$ and specificity $(86.7 \%)$, with a PPV $=80 \%$, an $\mathrm{NPV}=76.5 \%$, and accuracy $=77.8 \%$ to predict the outcome (dead vs. alive at EOF).

\section{NEP-D threshold}

A NEP-D score threshold was investigated to predict survival. We found that a value of NEP-D $\geq 90$ represents a cutoff, allowing the best compromise between sensitivity $(67 \%)$ and specificity (67\%), with a PPV $=61 \%$, a NPV $=71 \%$, and accuracy $=82 \%$ to predict the outcome (dead vs. alive at EOF). Indeed, at EOF, $61.5 \%$ of patients with NEP-D $\geq 90$ and $28.5 \%$ of patients with NEP-D $<90$ were dead. However, statistical evaluation did not show any significance. Mean OS of patients dead at EOF was 47.5 months; in patients with NEP-D $<90$ mean OS was $90 \pm 33$ months, while in patients with NEP-D $\geq 90$ mean OS was significantly shorter, corresponding to $26.2 \pm 8.5$ months (Fig. $3 \mathrm{a} ; p<0.05$ ).

On the other hand, a value of NEP-D $\geq 116$ displays a moderate sensitivity (58\%) with a specificity of $80 \%$, $\mathrm{PPV}=70 \%, \mathrm{NPV}=71 \%$, and accuracy $=70 \%$ to predict the outcome (dead vs. alive at the EOF). At EOF $70 \%$ of patients with NEP-D $\geq 116$ and $29.5 \%$ of patients with NEP-D $<116$

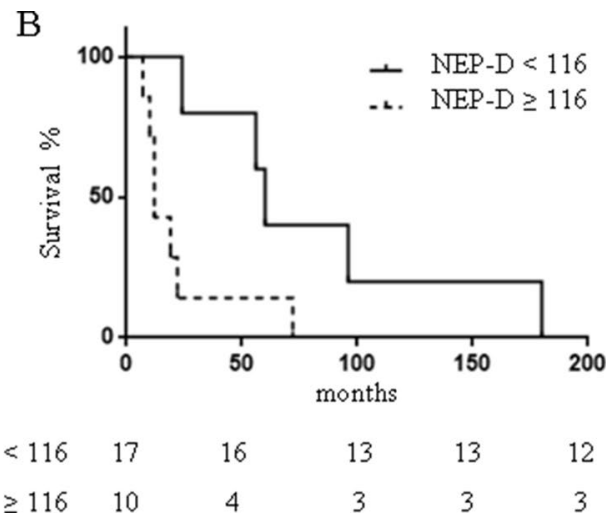

(continuous line) with $\mathrm{OS}=90 \pm 33$ months or $\geq 90$ (dotted line) with OS $=26.2 \pm 8.5$ months $(p<0.05)$. b Survival curve of patients with NEP-D $<116$ (continuous line) with $\mathrm{OS}=83.2 \pm 26.8$ months or $\geq 116$ (dotted line) with OS $=22.0 \pm 8.6$ months $(p<0.05)$ 
were dead $(\mathrm{p}<0.05)$. In patients with NEP-D $<116$ mean OS was $83.2 \pm 26.8$ months, while in patients with NEP-D $\geq 116$ mean OS was significantly shorter $(22.0 \pm 8.6$ months; $p<0.05$ ) (Fig. 3b).

Moreover, we calculated the difference between NEP-T and NEP-D for each patient as a "Delta-NEP score". In patients who were alive at EOF mean Delta-NEP score was $13 \pm 7.2$, while in patients who were dead at EOF mean Delta-NEP score was significantly higher $(56 \pm 8.2$; $p$ value $<0.05)$.

\section{Patients' survival}

During follow-up ( $70.3 \pm 11.6$ months $), 12$ patients died due to disease progression, with an overall mortality of $44.4 \%$ and a mean OS $=51.2 \pm 14.9$ month. We investigated whether the identified NEP-D thresholds may be useful to predict 5 years survival: we found that 5 out of 6 patients with NEP-D score $<90$ were alive, while 4 out of 11 patients with NEP-D score $\geq 90$ were alive 5 years after diagnosis. Similar results were found with the NEP-D threshold set at 116: 7 out of 12 patients with NEP-D score $<116$ and 2 out of 9 patients with NEP-D score $\geq 116$ were alive 5 years after diagnosis. However, the observed different distribution did not reach statistical significance in both cases, probably due to the low patient number.

When evaluating mean Delta-NEP score, we found that in patients with ileal NEN $(38.7 \pm 10.3)$, this score was not significantly different from that of patients with pancreatic NEN (pNEN) (36.2 \pm 9.2$)$. On the other hand, patients with ileal NEN showed a sixfold longer mean OS as compared to patients with pNEN $(81.3 \pm 21.9$ vs. $13.6 \pm 2.3$ month; $p<0.05$ ) in keeping with previous reports [16].

\section{Discussion}

Biological behavior of GEP NEN is very heterogeneous and several factors associated with both the patient and the tumor may modify prognosis. The possibility of identifying a score that could predict GEP NEN patients' prognosis is of great interest for clinicians. Despite that the majority of GEP NEN are characterized by an indolent clinical course, a subgroup displays an unpredictable behavior, characterized by an unfavorable outcome and worse survival as compared to patients with the same characteristics. Patients with WD stage IV disease may be treated by several approaches, including somatostatin analogs (SSA), chemotherapy, peptide receptor radionuclide therapy (PRRT), and targeted therapies, even though a sequence has not been precisely established so far [6, 15, 23-25]. However, these approaches may temporarily control disease progression, which frequently follows the first therapeutic line $[6,15]$. Therefore, it would be very important to identify those patients that may benefit by an aggressive treatment from those who could be spared from unnecessary therapy. Consolidated prognostic factors are represented by morphology (i.e., differentiation), proliferation rate in terms of number of mitosis and proliferation index [5, 7, 10, 13, 14, 26, 27]. Currently, Ki67 is considered one of the most important prognostic factors in NEN [5, 7, $14,26,28]$. However, useful criteria to subdivide stage IV GEP NEN are not available to precisely assess prognosis and metastatic burden value.

Classification systems are continuously updated, contributing to a better identification of prognostic characteristics of each NEN category. However, few studies have investigated prognostic indexes capable of estimating survival in stage IV GEP NEN. The prognostic NEP-Score identified by Pusceddu et al. was found to be useful to stratify survival probability also in very heterogeneous patients groups, but could be improved by including additional information, such as biochemical [8, 9, 11, 12, 17], molecular [5, 19, 29-32] and immunohistochemical markers [20,21], imaging characteristics [33, 34], gender [8, 9, 35, 37], ethnicity [8, 9], nodal involvement, tumor size [8, 9, 11, 12, 28, 35], previous treatments [8,9], and metastatic disease burden $[8,9,11,16]$. In addition, the influence of medical treatment outcome and of therapeutic advances developed during the last years [23-25, 36] were not taken into account when considering OS.

In our study, we validated the NEP-Score in an independent homogeneous series of 27 patients with stage IV WD entero-pancreatic NEN followed up in our center from 1998 to 2018 . We confirm the validity of the score, since our NEP-T, corresponding to the NEP-Score in the study by Pusceddu et al., was found to be higher in patients dead as compared to patients alive at EOF. This finding confirms that NEP-T score can be adopted in very different clinical settings. However, its utility is impaired by the fact that a 24 months follow-up is needed to calculate the score. We therefore modified the score, taking into account only the characteristics available at diagnosis, obtaining the NEP-D score that does not consider the development of metachronous metastases. We then investigated whether NEP-D may predict survival, also evaluating gender differences. We found that NEP-D is significantly higher in male patients that are dead as compared to those alive at EOF, independently of age. This finding was not replicated in females, probably due to the low subject number in the group of patients dead at EOF (only three patients). In the group of patients alive at EOF, males displayed a significantly lower mean NEP-D as compared to females, probably due to a greater prevalence in the latter group of pNEN, a primary site associated with a greater score as compared to ileal NEN. In the group of patients dead at EOF, no significant differences were found between genders in terms of NEP-D. These results indicate that 
NEP-D does not depend on patients' gender, suggesting that stage IV NEN entero-pancreatic patients prognosis is not influenced by gender. This hypothesis is further strengthened by the evidence that OS is similar in both genders. Our data are not in line with previous evidence reporting a protective role for female gender in patients aged $>75$ years with pNEN [37]. This difference may be due to patient selection in our study, which includes subjects with a wider age range (26-84 years) with only five patients $>75$ years $(2 \mathrm{~F}$ and $3 \mathrm{M})$. Therefore, these studies cannot be compared.

The prognostic significance of metastatic disease burden and progression at metastatic sites is difficult to assess. The study by Pusceddu et al. allows to quantify this item by assigning a score that we validated in our cohort. We then developed a score that does not depend on this item, and set out to identify a threshold that could predict survival since the diagnosis. The identified thresholds have slightly different performance, with an NEP-D of 90 representing the best compromise between sensitivity and specificity. However, this threshold did not reach statistical significance, probably due to the low number of investigated patients. This does not hold true for the NEP-D threshold of 116 that, however, displays a worse sensitivity. Therefore, this threshold may underestimate NEP-D value as a marker of survival. On the other hand, they may be useful to predict 5 years survival at the time of diagnosis, providing further help to plan a patient-tailored management. However, these thresholds need to be validated in independent cohorts of patients with stage IV entero-pancreatic NEN.

Delta-NEP score may offer more indications, since it measures the development of new metastases, with a different score depending on the timing (before or after 24 months from diagnosis). The evidence that this score is significantly higher in patients dead as compared to those alive at EOF suggests that metastases development in new sites profoundly influences survival in stage IV NEN GEP patients, being more important than the progression of metastases already present at diagnosis. The evidence that ileal and pNEN do not differ concerning this parameter suggests that metastatic disease progression was similar in the two groups and confirms the pancreatic site as an independent unfavorable prognostic marker. This hypothesis is further strengthened by the evidence that patients with ileal NEN showed a sixfold longer mean OS as compared to patients' pNEN, in keeping with previous reports [16].

In conclusion, our study suggests NEP-D as a simple, quick, cheap, easy to calculate prognostic score that employs information readily available and, at the same time, provides help to clinicians in decision making. This score encloses in a single index the characteristics known to influence prognosis in GEP NEN (grade, stage, age at diagnosis, primary site) [7-12, 16, 26, 27].

NEP-D application could predict the aggressiveness of each NEN to better identify those patients that will benefit from an aggressive and timely treatment as well as those with an indolent disease that could be spared from overtreatment.

Acknowledgements We would like to thank our colleagues who provided advice in the multidisciplinary team that takes care of NEN patients at the Azienda Ospedaliero-Universitaria di Ferrara; Via Aldo Moro 8, 44124 Cona-Ferrara, Italy.

Funding This research was in part supported by funds of the Italian Ministry of Education, University and Research (PRIN 2017Z3N3YC). Open access funding provided by Università degli Studi di Ferrara within the CRUI-CARE Agreement.

\section{Compliance with ethical standards}

Conflict of interest The authors declare that the research was conducted in the absence of any commercial or financial relationships that could be construed as a potential conflict of interest.

Ethical approval This study is in accordance with the principles set out in the Declaration of Helsinki, has been specifically approved by the Local Ethics Committee (Comitato Etico Indipendente di Area Vasta Emilia Centro, CE-AVEC, at the Policlinico S.Orsola-Malpighi in Bologna) and authorized by the General Director of the Azienda Ospedaliero Universitaria in Ferrara (protocol number CE-AVEC 238/2020/Oss/AOUFe).

Informed consent Written consent was obtained from each patient or subject after full explanation of the purpose and nature of all procedures used.

Open Access This article is licensed under a Creative Commons Attribution 4.0 International License, which permits use, sharing, adaptation, distribution and reproduction in any medium or format, as long as you give appropriate credit to the original author(s) and the source, provide a link to the Creative Commons licence, and indicate if changes were made. The images or other third party material in this article are included in the article's Creative Commons licence, unless indicated otherwise in a credit line to the material. If material is not included in the article's Creative Commons licence and your intended use is not permitted by statutory regulation or exceeds the permitted use, you will need to obtain permission directly from the copyright holder. To view a copy of this licence, visit http://creativecommons.org/licenses/by/4.0/.

\section{References}

1. Dasari A, Shen C, Halperin D, Zhao B, Zhou S, Xu Y, Shih T, Yao JC (2017) Trends in the incidence, prevalence, and survival outcomes in patients with neuroendocrine tumors in the United States. JAMA Oncol 3:1335-1342

2. Sackstein PE, O'Neil DS, Neugut AI, Chabot J, Fojo T (2018) Epidemiologic trends in neuroendocrine tumors: an examination of incidence rates and survival of specific patient subgroups over the past 20 years. Semin Oncol 45:249-258. https://doi. org/10.1053/j.seminoncol.2018.07.001 
3. Fraenkel M, Kim MK, Faggiano A, Valk GD (2012) Epidemiology of gastroenteropancreatic neuroendocrine tumours. Best Pract Res Clin Gastroenterol 26:691-703

4. Lawrence B, Gustafsson BI, Chan A, Svejda B, Kidd M, Modlin IM (2011) The epidemiology of gastroenteropancreatic neuroendocrine tumors. Endocrinol Metab Clin North Am 40:1-18

5. Guilmette JM, Nosé V (2019) Neoplasms of the neuroendocrine pancreas: an update in the classification, definition, and molecular genetic advances. Adv Anatom Pathol 26:13-30. https://doi. org/10.1097/PAP.0000000000000201

6. Oberg K, Knigge U, Kwekkeboom D, Perren A (2012) Neuroendocrine gastro-entero-pancreatic tumors: ESMO Clinical Practice Guidelines for diagnosis, treatment and follow-up. Ann Oncol 23(Suppl 7):vii124-vii130

7. Rindi G, Falconi M, Klersy C, Albarello L, Boninsegna L, Buchler MW, Capella C, Caplin M, Couvelard A, Doglioni C, Delle Fave G, Fischer L, Fusai G, de Herder WW, Jann H, Komminoth P, de Krijger RR, La Rosa S, Luong TV, Pape U, Perren A, Ruszniewski P, Scarpa A, Schmitt A, Solcia E, Wiedenmann B (2012) TNM staging of neoplasms of the endocrine pancreas: results from a large international cohort study. J Nat Cancer Inst 104:764-777. https://doi.org/10.1093/jnci/djs208

8. Modlin IM, Gustafsson BI, Pavel M, Svejda B, Lawrence B, Kidd M (2010) A nomogram to assess small-intestinal neuroendocrine tumor ("Carcinoid") survival. Neuroendocrinology 92:143-157. https://doi.org/10.1159/000319784

9. Kelly S, Aalberg J, Agathis A, Phillips K, Haile S, Haines K, Kang Kim M, Divino CM (2019) Predicting survival of small intestine neuroendocrine tumors: experience from a major referral center. Pancreas 48:514-518. https://doi.org/10.1097/MPA.0000000000 001296

10. Kloppel G, Rindi G, Perren A, Komminoth PK, DS, (2010) The ENETS and AJCC/UICC TNM classifications of the neuroendocrine tumors of the gastrointestinal tract and the pancreas: a statement. Virchows Arch 456:595-597. https://doi.org/10.1007/ s00428-010-0924-6

11. Pape UF, Berndt U, Muller-Nordhorn J, Bohmig M, Roll S, Koch M, Willich SN, Wiedenmann B (2008) Prognostic factors of longterm outcome in gastroenteropancreatic neuroendocrine tumours. Endocr Relat Cancer 15:1083-1097. https://doi.org/10.1677/ ERC-08-0017

12. Durante C, Boukheris H, Dromain C, Duvillard P, Leboulleux S, Elias D, de Baere T, Malka D, Lumbroso J, Guigay J, Schlumberger M, Ducreux M, Baudin E (2009) Prognostic factors influencing survival from metastatic (stage IV) gastroenteropancreatic well-differentiated endocrine carcinoma. Endocr Relat Cancer 16:585-597. https://doi.org/10.1677/ERC-08-0301

13. Rindi G, Klersy C, Albarello L, Baudin E, Bianchi A, Buchler MW, Caplin M, Couvelard A, Cros J, de Herder WW, Delle Fave G, Doglioni C, Federspiel B, Fischer L, Fusai G, Gavazzi F, Hansen CP, Inzani F, Jann H, Komminoth P, Knigge UP, Landoni L, La Rosa S, Lawlor RT, Luong TV, Marinoni I, Panzuto F, Pape UF, Partelli S, Perren A, Rinzivillo M, Rubini C, Ruszniewski P, Scarpa A, Schmitt A, Schinzari G, Scoazec JY, Sessa F, Solcia E, Spaggiari P, Toumpanakis C, Vanoli A, Wiedenmann B, Zamboni G, Zandee WT, Zerbi A, Falconi M (2018) Competitive testing of the WHO 2010 versus the WHO 2017 grading of pancreatic neuroendocrine neoplasms: data from a large international cohort study. Neuroendocrinology 107:375-386. https:// doi.org/10.1159/000494355

14. Rindi G, Petrone G, Inzani F (2014) The 2010 WHO classification of digestive neuroendocrine neoplasms: a critical appraisal four years after its introduction. Endocr Pathol 25:186-192. https://doi. org/10.1007/s12022-014-9313-Z
15. Pavel M, O'Toole D, Costa F, Capdevila J, Gross D, Kianmanesh R, Krenning E, Knigge U, Salazar R, Pape UF, Öberg K (2016) ENETS Consensus Guidelines update for the management of distant metastatic disease of intestinal, pancreatic, bronchial neuroendocrine neoplasms (NEN) and NEN of unknown primary site. Neuroendocrinology 103:172-185. https://doi.org/10.1159/00044 3167

16. Panzuto F, Merola E, Pavel ME, Rinke A, Kump P, Partelli S, Rinzivillo M, Rodriguez-Laval V, Pape UF, Lipp R, Gress T, Wiedenmann B, Falconi M, Delle Fave G (2017) Stage IV gastro-entero-pancreatic neuroendocrine neoplasms: a risk score to predict clinical outcome. Oncologist 22:409-415. https://doi. org/10.1634/theoncologist.2016-0351

17. Marotta V, Zatelli MC, Sciammarella C, Ambrosio MR, Bondanelli M, Colao A, Faggiano A (2018) Chromogranin A as circulating marker for diagnosis and management of neuroendocrine neoplasms: more flaws than fame. Endocr Relat Cancer. 25:R11R29. https://doi.org/10.1530/ERC-17-0269

18. Arnold R, Wilke A, Rinke A, Mayer C, Kann PH, Klose KJ, Scherag A, Hahmann M, Müller HH, Barth P (2008) Plasma chromogranin $\mathrm{A}$ as marker for survival in patients with metastatic endocrine gastroenteropancreatic tumors. Clin Gastroenterol Hepatol 6:820-827. https://doi.org/10.1016/j.cgh.2008.02.052

19. Zatelli MC, Grossrubatscher EM, Guadagno E, Sciammarella C, Faggiano A, Colao A (2017) Circulating tumor cells and miRNAs as prognostic markers in neuroendocrine neoplasms. Endocr Relat Cancer 24:R223-R237. https://doi.org/10.1530/ERC-17-0091

20. Zatelli MC, Fanciulli G, Malandrino P, Ramundo V, Faggiano A, Colao A, NIKE Group (2016) Predictive factors of response to mTOR inhibitors in neuroendocrine tumours. Endocr Relat Cancer. 23(3):R173-R183. https://doi.org/10.1530/ERC-15-0413

21. Viúdez A, Carvalho FL, Maleki Z, Zahurak M, Laheru D, Stark A, Azad NS, Wolfgang CL, Baylin S, Herman JG, De Jesus-Acosta A (2016) A new immunohistochemistry prognostic score (IPS) for recurrence and survival in resected pancreatic neuroendocrine tumors (PanNET). Oncotarget. 7(18):24950-24961. https://doi. org/10.18632/oncotarget.7436

22. Pusceddu S, Barretta F, Trama A, Botta L, Milione M, Buzzoni R, De Braud F, Mazzaferro V, Pastorino U, Seregni E, Mariani L, Gatta G, Di Bartolomeo M, Femia D, Prinzi N, Coppa J, Panzuto F, Antonuzzo L, Bajetta E, Brizzi MP, Campana D, Catena L, Comber H, Dwane F, Fazio N, Faggiano A, Giuffrida D, Henau K, Ibrahim T, Marconcini R, Massironi S, Žakelj MP, Spada F, Tafuto S, Van Eycken E, Van der Zwan JM, Žagar T, Giacomelli L, Miceli R, NEPscore Working Group (2018) A classification prognostic score to predict OS in stage IV well-differentiated neuroendocrine tumors. Endocr Relat Cancer 25:607-618. https://doi. org/10.1530/ERC-17-0489

23. Garcia-Carbonero R, Rinke A, Valle JW, Fazio N, Caplin M, Gorbounova V, O'Connor J, Eriksson B, Sorbye H, Kulke M, Chen J, Falkerby J, Costa F, de Herder W, Lombard-Bohas C, Pavel M (2017) ENETS consensus guidelines for the standards of care in neuroendocrine neoplasms: systemic therapy—chemotherapy. Neuroendocrinology 105:281-294. https://doi.org/10.1159/00047 3892

24. Pavel M, Valle JW, Eriksson B, Rinke A, Caplin M, Chen J, Costa F, Falkerby J, Fazio N, Gorbounova V, de Herder W, Kulke M, Lombard-Bohas C, O'Connor J, Sorbye H, Garcia-Carbonero R (2017) ENETS consensus guidelines for the standards of care in neuroendocrine neoplasms: systemic therapy—biotherapy and novel targeted agents. Neuroendocrinology 105:266-280. https ://doi.org/10.1159/000471880

25. Hicks RJ, Kwekkeboom DJ, Krenning E, Bodei L, GrozinskyGlasberg S, Arnold R, Borbath I, Cwikla J, Toumpanakis C, Kaltsas G, Davies P, Hörsch D, Tiensuu Janson E, Ramage J 
(2017) ENETS consensus guidelines for the standards of care in neuroendocrine neoplasms: peptide receptor radionuclide therapy with radiolabelled somatostatin analogues. Neuroendocrinology 105:295-309. https://doi.org/10.1159/000475526

26. Klöppel G, La Rosa S (2018) Ki67 labeling index: assessment and prognostic role in gastroenteropancreatic neuroendocrine neoplasms. Virchows Arch 472:341-349. https://doi.org/10.1007/ s00428-017-2258-0

27. Inzani F, Petrone G, Rindi G (2018) The New World Health Organization classification for pancreatic neuroendocrine neoplasia. Endocrinol Metab Clin N Am 47:463-470. https://doi. org/10.1016/j.ecl.2018.04.008

28. Dima SO, Dumitrascu T, Pechianu C, Grigorie RT, Brasoveanu V, Sorop A, Lupescu I, Purnichescu-Purtan R, Croitoru A, Bacalbasa N, Tanase A et al (2018) Prognostic factors in patients with surgical resection of pancreatic neuroendocrine tumours. Acta Endocrinol (Buchar). 14:389-393. https://doi.org/10.4183/ aeb.2018.389

29. Scarpa A, Chang DK, Nones K, Corbo V, Patch AM, Bailey P, Lawlor RT, Johns AL, Miller DK, Mafficini A (2017) Wholegenome landscape of pancreatic neuroendocrine tumours. Nature 543:65-71. https://doi.org/10.1038/nature21063

30. Malczewska A, Witkowska M, Makulik K, Bocian A, Walter A, Pilch-Kowalczyk J, Zajęcki W, Bodei L, Oberg KE, Kos-Kudła B (2019) NETest liquid biopsy is diagnostic of small intestine and pancreatic neuroendocrine tumors and correlates with imaging. Endocr Connect 8:442-453. https://doi.org/10.1530/EC-19-0030

31. Malczewska A, Bodei L, Kidd M, Modlin IM (2019) Blood mRNA measurement (NETest) for neuroendocrine tumor diagnosis of image-negative liver metastatic disease. J Clin Endocrinol Metab 104:867-872. https://doi.org/10.1210/jc.2018-01804

32. Modlin IM, Kidd M, Malczewska A, Drozdov I, Bodei L, Matar S, Chung KM (2018) The NETest: the clinical utility of multigene blood analysis in the diagnosis and management of neuroendocrine tumors. Endocrinol Metab Clin North Am 47:485-504. https ://doi.org/10.1016/j.ecl.2018.05.002.Review

33. Hindié E (2017) The NETPET score: combining FDG and somatostatin receptor imaging for optimal management of patients with metastatic well-differentiated neuroendocrine tumors. Theranostics 7:1159-1163. https://doi.org/10.7150/thno.19588

34. Chan DL, Pavlakis N, Schembri GP, Bernard EJ, Hsiao E, Hayes A, Barnes T, Diakos C, Khasraw M, Samra J, Eslick E, Roach PJ, Engel A, Clarke SJ, Bailey DL (2017) Dual somatostatin receptor/FDG PET/CT imaging in metastatic neuroendocrine tumours: proposal for a novel grading scheme with prognostic significance. Theranostics 7:1149-1158. https://doi.org/10.7150/thno.18068

35. Panzuto F, Campana D, Massironi S, Faggiano A, Rinzivillo M, Lamberti G, Sciola V, Lahner E, Manuzzi L, Colao A, Annibale B (2019) Tumour type and size are prognostic factors in gastric neuroendocrine neoplasia: a multicentre retrospective study. Dig Liver Dis. https://doi.org/10.1016/j.dld.2019.04.016

36. Zandee WT, de Herder WW (2018) The evolution of neuroendocrine tumor treatment reflected by ENETS guidelines. Neuroendocrinology 106:357-365. https://doi.org/10.1159/000486096

37. Li G, Tian ML, Bing YT, Tao LY, Wang HY, Jiang B, Yuan CH, Xiu DR (2019) Clinicopathological features and prognosis factors for survival in elderly patients with pancreatic neuroendocrine tumor: A STROBE-compliant article. Medicine (Baltimore) 98(11):e14576. https://doi.org/10.1097/MD.0000000000014576

Publisher's Note Springer Nature remains neutral with regard to jurisdictional claims in published maps and institutional affiliations. 